\title{
COSMOLOGICAL MODELS: THEORY AND OBSERVATIONS
}

\author{
V.N.Lukash ${ }^{1}$ \\ ${ }^{1}$ Astro Space Center of Lebedev Physical Institute (ASC FIAN), \\ 84/32 Profsoyuznaya st, Moscow, 117810, Russia
}

\begin{abstract}
The current status of the theoretical and observational cosmology is reviewed.

\section{INTRODUCTION}

Investigation of the anisotropy of the relic CMB radiation ensures a basic channel of the information about the early Universe. The continuing progress in the technology of deep-sky galactic surveys has resulted in impressive knowledge on the Large Scale Structure in the Universe as well as its evolution back to high redshifts. Both $\Delta T / T$ and LSS observational data complement on supercluster scales and thus disclose the cosmological model. Geometrical experiments (SNIa, Deuterium) provide additional independent tests hinting on a complex nature of the dark matter in the Universe.
\end{abstract}

The cosmological model gets parameters. There is a persistent evidence that

- the underlying space is flat,

- the Hubble parameter is close to $65 \mathrm{~km} \mathrm{~s}^{-1} \mathrm{Mpc}^{-1}$ which is consistent with the age of old globular clusters if $\Omega_{m} \leq 0.5$,

- the structure in the Universe has developed in the process of gravitational instability from small Gaussian perturbations of matter density with a scale invariant fundamental spectrum.

The situation in cosmology is currently dominated by observations, however the accuracy of present observational data is still too low to determine a unique set of all cosmological parameters. The main physical issue - a nature and composition of dark matter - is still under discussion. A great hope of cosmologists is related with the development of ground and space based $\Delta T / T$ and far Universe observations which will help to delimit the cosmological model up to a few per cent in the nearest future.

\section{FORMATION OF LSS IN THE UNIVERSE}

The seeds of the visible large scale structure in the Universe are the Cosmological Density Perturbations which grow due to gravitational instability in the late period of the Universe expasion when density is dominated by cold non-relativistic matter $\left(z_{\sim}^{<} 10^{4}\right)$. These primordial CDPs must have been created at the inflationary Big Bang epoch, as after the end of inflation the Universe was hot (radiationally dominated) and thus gravitationally stable against small perturbations of matter density and gravitaty field. The CDPs existed in the hot Universe as longwave 'gravitating sound waves' propagating across the relativistic matter with a constant amplitude.

The CDPs started growing only after the equality epoch when the matter pressure decayed. The required fundamental CDP for galaxy clusters to be formed by now, has therefore been predicted at the level $\delta \sim$ $3 \times 10^{-5}$ which was finally confirmed by COBE (Bennett et al., 1996) and BOOMERanG (Jaffe et al., 2000) on this same level but at larger scales (that in turn appeared to be in a successful consistency with another famous prediction known as the Harrison-Zel'dovich scale invariant perturbation spectrum). At the same 
time, the CDP amplitude at Mpc scale should have been enhanced as indicated by $L y_{\alpha}$-forest and early galaxy formation. This can be achieved by introducing weakly interacting massive particles (Cold Dark Matter): contrary to baryionic damping, the density contrast of cold collisionless particles grows logarithmically in the hot Universe boosting the power spectrum at galactic scales $\left(k>k_{e q} \simeq 0.05 h \mathrm{Mpc}^{-1}\right)$.

Such an optimistic situation in understanding the structure formation has produced a strong impetus for the observational and theoretical cosmology extending dramatically by continuing progress in the improved technology of deep-sky surveys and CMB temperature detections. The ultimate goal is to reconstruct the model parameters and CDP power spectrum from Mpc up to the horizon scale, the scope straighforwardly related to the high energy physics thus capable of being observationally tested now.

Three points should be emphasised in connection with the problem of LSS formation: theoretical, model, and observational.

The first point means that LSS formation in the Universe is as fundamental problem as the creation of the Universe as a whole: both features, the small CDPs and Friedmannian background model (the Cosmological Principle), were produced in a unique process of inflation in the very early Universe. The theory works at very high energies $\left(\sim 10^{13} \mathrm{GeV}\right)$ whereas the observations occur in a low-energy limit $\left(\sim 10^{-4} \mathrm{eV}\right)$. To provide a fair comparison in such a situation we need a model to know how the perturbations evolved during the entire history of the Universe. Therefore, any confrontation in cosmology between theory and observations appears to be model dependent.

To determine the model we need priorities and parameters. The former assumes gravitational instability as the principal mechanism of CDP dynamics at large scales, and spatially ramdom Gaussian primordial perturbations (both assumptions stay in good agreement with observations). The latter assumes knowledge of the current time when the structure is observed $\left(H_{0}\right)$, the composition and abundance of cosmic matter $\left(\Omega_{m}=\Omega_{c}+\Omega_{\nu}+\Omega_{b}\right)$, dark energy $\left(\Omega_{\Lambda}\right.$, quintessence, relic scalar field), Cosmic Gravitational Waves $(\mathrm{T} / \mathrm{S})$, the number of species of massive neutrinos and relativistic particles, the parameters of the perturbation spectra $\left(n_{S}, n_{T}, \sigma_{8}\right)$ and biasing coefficients $\left(b_{c l}, b_{g a l}\right)$, the optical depth at reheating, etc. Nowadays cosmologists venture the following approach: if a family of the dark matter models is postulated as fairly simple (with a few model parameters) then the recovering of both the CDP power spectrum and the cosmological parameters can be provided by a given set of observational data.

Below, we discuss the model under such a conventional probability sense. There is no principal restrictions on this way: any theory can be tested to the limit if we have enough data. The more data are available the less uncertainties remain in the theory and more parameters can be determined. Actually, we are now in the beginning of data collection. Cosmologists have started the model restoration exercise taking simple theories and confronting them with the observational data available. This development progresses with an increasing number of model parameters. Theory goes from simplicity, however Nature appears complex.

\section{DARK MATTER MODELS}

Until recently there were two basic theories claiming to approach the corner stones of the LSS formation: inflation and defects. While being very much different in their grounds on galaxy seeds - the linear Gaussian scalar perturbations in one case and the non-linear non-Gaussian cosmic defects (strings, monopoles, textures) in other case - both models presented the fundamental inevitable perturbations produced in the very early Universe: the parametrically amplified quantum vacuum fluctuations of the inflaton and the topological defects left after phase transitions in the early Universe, respectively.

However, a simple defect models normalised by the CMB fluctuations have failed to meet the LSS formation (Watson, 1997). The reason is that the non-linear matter perturbations generate all three types of the metric fluctuations (scalar, vortex and tensor) which all contribute to the Sachs-Wolfe $\Delta T / T$ anisotropy on large angular scale, so the resulting S-mode amplitude appears insufficient to develop the observed galaxy distribution.

By now only the inflation theories (adiabatic Gaussian density perturbations) with cold dark matter have got through ordeals of fitting the LSS and $\Delta T / T$ requirements. The principal quest here is the predicted Gaussian nature of small CDPs, which faces a satisfactory consistency with the real distribution of galaxies

on scales $\sim 20 h^{-1} \mathrm{Mpc}$ (e.g. Juszkiewicz and Bouchet, 1996). The only obstacle to test reliably this important feature of the CDP seeds is the restricted depth of the available galaxy surveys. 
Deep galaxy surveys would also be highly welcome for clarifying another challenge of the modern cosmology: the fractal model attacking persistently the cosmological principle. The point is that large voids seen in the field galaxy and cluster distributions extend up to scales $\sim 100 h^{-1} \mathrm{Mpc}$ which is close to catalogues' sizes, thus leaving a room for discussions about the value of the homogeneity scale (Sylos-Labini et al., 1997). Nevertheless, it sould be emphasized that the fractal challenge is rather an issue for optical galaxies, and not for the distribution of the total mass in the Universe. The latter should be pretty homogeneous on scales larger than $\sim 10 h^{-1} \mathrm{Mpc}$ to fit beautiful Hubble diagrams, to say nothing on the uniform microwave and X-ray backgrounds evidencing the cosmic homogeneity at larger scales.

Thus, we consider only models backed by inflationary theories. The main tool for Gaussian perturbations is the second moment of their spatial distribution related to the power spectrum:

$\left\langle\delta^{2}\right\rangle=\int_{0}^{\infty} P(k) k^{3} d k=\int_{0}^{\infty} \Delta_{k}^{2} \frac{d k}{k}$.

The dimensionless CDP spectrum $\Delta_{k}^{2}$ has a simple meaning of the variance of density contrast in the scale $k$ (the wave number) within the scale band $d k \sim k$, it is evidently additive $\left(\delta^{2} \sim \Sigma \Delta_{k}^{2}\right)$.

Before passing to the discussion on the spectrum observational reconstruction let me sketch briefly the situation with cosmological parameters.

\section{COSMOLOGICAL PARAMETERS}

It seems that the longstanding debate on $H_{0}=100 h \mathrm{~km} \mathrm{~s}^{-1} \mathrm{Mpc}^{-1}$ is approaching to its end and we are going to learn the value of the Hubble constant soon. Today, two methods are very promising: measuring Cepheids in distant galaxies and the supernovae type Ia. The current agreement stands for the value of $h$ between 0.6 and 0.7 with accuracy $\sim 10 \%$, which marginally excludes the matter dominated models with a negligible cosmological constant (such models would require $h<0.6$ ) regarding the low limit for the age of the Universe coming from globular clusters.

Another issue is the matter abundance in the Universe.

At the first glance the situation is a following: again we have two groups of experiment resulting in different conclusions. However, the agreement is possible.

The first experiment deals with megaparsec scales - galaxy halos, groups and X-ray clusters. An assumption on the hydrostatic equilibrium within cluster cores yields a low dynamical mass responsible for the shape of gravitational potential on Mpc scale: $\Omega_{m} \sim 0.3$. Another important observation is a large fraction of baryons inside X-ray clusters reaching somehow $\sim 20 \%$ within scale $\sim 1$ Mpc:

$\frac{M_{b}}{M_{m}} \sim 0.2$

which is also consistent with a low matter density involved dynamically in small scales: if $\Omega_{b} \leq 0.1$ due to the deuterium measurements, $\Omega_{m} \sim \Omega_{b}\left(M_{m} / M_{b}\right) \lesssim 0.5$ at the dynamical scale $\left(l_{D} \sim 10 h^{-1} \mathrm{Mpc}\right)$.

However, some experiments dealing with LSS $\left(l>l_{D}\right)$ hints at a matter domination $\left(\Omega_{m}>0.5\right)$. There are few arguments for it (still more model dependent ones in comparison with the small-scale arguments):

- the existence of substructures in the majority of galaxy clusters evidencing that the clusters are just forming systems, which is possible only in the Universe dynamically close to the critical density of matter;

- the large coherence bulk velocities obviously of the cosmological origin, allowing a principal reconstruction of the total density contrast (and, in some cases, the consistency with a model $\Omega_{m} \sim 0.5$ and the galaxy 'biasing' factor $b \sim 1$ );

- the weak gravitational lensing confirming high dynamical mass abundance around some X-ray clusters;

- the lensing argument on the fraction of splitting quasars (still much dependent on the model assumptions); 
- the high abundance of $L y_{\alpha}$ absorption systems (the $L y_{\alpha}$ clouds);

- the evolutionary argument on the galaxy clusters number density (still under discussion).

The low evolution argument about the dependence of cluster abundance on redshifts, which for many years was considered as a basic argument in favour of a low density Universe, is not any more as strong as it seemed before. It is the ENACS identification of the nearby galaxy clusters (by the dispersion velocities of their optical galaxies, see Mazure et al. (1996)) that has shown a previous underestimation of the number density of nearby Abell clusters. At the moment we may state the consistency of the cluster number density evolution curve with a model $\Omega_{m} \sim 0.5$.

A breakthrough in the problem of the model geometry restoration is being currently done while building up classical Hubble diagrams (redshifts vs apparent magnitudes) for distant supernovae of type Ia (Perlmutter at al., 1998). Contrary to galaxies, such sources look amazingly standard candles which is well supported by the distance measurements to nearby supernovae. Tested by distant supernovae Ia, the deviations of the Hubble diagram from the linear law hint upon the real geometry of the Universe. Currently, the predictions are close to a half-to-half matter/vacuum Universe $\left(\Omega_{m} \sim \Omega_{\Lambda} \sim 0.5\right)$. However, a careful analysis of the systematic effects is required to make this method really trustable.

While reconstructing cosmological parameters from the CMB temperature fluctuations we see the strongest effect coming from the locations and amplitudes of the acoustic peaks (the Sakharov oscillations). The results of BOOMERanG and MAXIMA-1 have specified the first and second acoustic peaks which favours a spatially flat Universe with high abundance of baryons: $\Omega_{b} h^{2} \simeq 0.03$ (Jaffe et al., 2000). However, better understanding of the cosmological model will come after carefull analysis of the $2 \mathrm{~d}$ and $3 \mathrm{~d}$ acoustic peaks which is a matter of the nearest future.

Finally, a possible reconciliation between the DM experiments on small and large scales can be the following: some fraction of dark matter in the Universe is distributed on large scales and is not involved in galaxy halos and groups.

How can it be arranged?

Today we have purely theoretical ideas on such a possibility. The most frequently discussed are the models with non-zero $\Lambda$-term $\left(\Omega_{\Lambda} \in(0.5,0.7)\right.$ ), mixed dark matter (cold+hot, with the hot particles like massive neutrinos with a few $\mathrm{eV}$ rest mass and the corresponding density parameter $\Omega_{\nu} \in(0.2,0.4)$ ), and a combination of both (the $\Lambda \mathrm{MDM}$ models). In all cases CDM particles form a dynamic structure on Mpc scales, while on large scales there is an additional contribution coming from light neutrinos or/and vacuum density (the $\Lambda$-term affects the cosmological expansion rate and effectively shifts the power spectrum of density perturbations towards large scales). A sceptical point concerning these and other cosmology models which are considered today as possible candidates for the real Universe is as follows: all of them are multiparameter (non-minimal) models; the more parameters are involved, the better the situation is with the data confrontation.

Does the latter tell us that we miss something important in our discussion on the formation of the Universe structure? May be. I can only conclude here saying than none of the models under discussion meets all the observational tests. Say, regarding previous examples, for $\Lambda \neq 0$ models one can expect a large fraction of old (relaxed) galaxy clusters and lensed quasars, whereas the MDM models require $H_{0}<60 \mathrm{~km} \mathrm{~s}^{-1} \mathrm{Mpc}^{-1}$ and too a small abundance of X-ray clusters and high-redshift quasars. Not to speak that none of the models considered now can explain a sharp turn of the spectrum slope at $k \sim 0.05 h \mathrm{Mpc}^{-1}$ indicated by galaxy clusters' distribution in space. Probably, the dark matter has a more complex nature than we think, existing in the form of a relic scalar field left after inflation, or varing vacuun density, or some other form of interacting relic particles which requires special investigation. In such a situation the observational verifications become extremely important. The principal test here is the LSS evolution in the early Universe.

\section{THE SPECTRUM OF DENSITY PERTURBATIONS}

The cosmological models of LSS formation discussed today are aimed to fit the observational data at $z=0$. Thus we cannot distinguish between the models without going into their evolution at medium and high redshifts where the models demonstrate their essential difference.

Two main experiments promote a snowball progress in the reconstruction of the CDP spectrum, which 
was impossible in previous years: $\Delta T / T\left(\theta>1^{\prime}\right)$ and direct investigation of the evolution and hierarchy of LSSs. The reason for stimulating such a progress is that these two experiments confront each other and overlap: the $\Delta T / T$ investigations go nowadays to small comoving scales up to $l \sim 10 h^{-1} \mathrm{Mpc}$ (recall the corresponding angular scale in $\operatorname{arcmin} \theta \sim l h)$, and, at the same time, we observe a developed structure of clusters, filaments, voids, and superclusters reaching the scales $\sim 100 h^{-1} \mathrm{Mpc}$.

Any reasonable assumption on the "formation" of large voids and superclusters in Gaussian perturbation theories inevitably leads to $\Delta T / T$ predictions at $\sim 1^{0}$ capable of current detection. It is a puzzle that namely this scale specifies the horizon at the decoupling era and therefore the angular scale of the first acoustic peak.

The existence of the acoustic peaks was predicted by theory long ago. Now, the time for observations has come: the first peak is detected. The time is close when the rest peaks will be found which allow to precisely formulate and delimit the theory.

We are aware of the cosmological temperature anisotropy on large and small scales, and have some information on the whole spectrum of the CMB fluctuations. Fortunately, the small angular scales $\left(\theta<1^{0}\right)$ can be effectively tested from the Earth's surface. A hope is that the terrestrial instruments together with balloon ones as well as the MAP and Planck Surveyor satellites, will provide a sensitivity advance sufficient for the cosmological model reconstruction.

Meanwhile, the situation with the CDP spectrum looks rather dramatic. At large scales $\left(\sim 1000 h^{-1}\right.$ $\mathrm{Mpc}$ ) the fundamental spectrum is small in amplitude and consistent with the HZ slope:

$\Delta_{k}^{2} \sim k^{3+n_{S}}, \quad n_{S}=1.1 \pm 0.1$.

However, at smaller scales $\left(\leq 100 h^{-1} \mathrm{Mpc}\right.$ ) the power should be boosted as we observe there rich structures in spatial distribution of galaxies, clusters, $L y_{\alpha}$ systems, and distant sources like quasars.

The latter is especially important.

We live in the period of the decay of quasar and star formation activities (Boyle and Terlevich, 1997). We thus have a unique opportunity to observe these numerous early sources tracing the past dynamics of LSS formation. This would be extremely informative as the LSS perturbation amplitude, being still less than unity today at $l \sim 100 h^{-1} \mathrm{Mpc}$, was ever lower in the past, which would predict a strong inverse evolution of such huge systems as superclusters and voids forming due to the gravitational instability.

It seems that quasars, the active galactic nuclei of distant galaxies, form the LSS at medium redshifts $(z \sim 1-2)$ which is provided by their correlation function and the existence of large QSO groups recalling in properties (the comoving size and abundance) the local superclusters (Komberg et al., 1996). Actually, distant bright quasars may originate in merging galaxies in protoclusters, and thus can trace the sites of enhanced matter density at medium and high redshifts analogous to how galaxy clusters trace them in the near space. The dynamical formation of these early LSSs suggests that the spectral amplitude at superclusters scale $\left(\sim 100 h^{-1} \mathrm{Mpc}\right)$ should be comparable and pretty close to that at cluster scale $\left(\sim 10 \mathrm{~h}^{-1}\right.$ $\mathrm{Mpc}$ ), i.e. the CDP spectrum is nearly flat between those scales (Komberg and Lukash, 1994):

$\Delta_{k}^{2} \sim k^{0.9 \pm 0.2}$.

This estimate for the spectrum shape is also backed by the local observations of galaxy and galaxy cluster distributions (Guzzo, 1991, Peacock, 1996, Einasto et al., 1997).

A strong break in the spectrum slope from the HZ asymptotic (3) to the flat part (4) should have happened at supercluster scale $\left(\sim 100 h^{-1} \mathrm{Mpc}\right)$ which is obviously a real feature of the fundamental CDP spectrum. This 'signature of the God' in the primordial spectrum demands its explanation in physics of the early Universe.

I cannot help mentioning another connection between the very early Universe and the primordial perturbations. This is a possibility to have high abundance of cosmic gravitational waves contributing to large-scale CMB anisotropy.

There are at least two reasons for such discussion.

The first one is theoretical. Inflation theory is not discriminative to any of the perturbation modes if inflation occurs at GUT energies (Lukash and Mikheeva, 1996, 2000): both S (CDP) and T (CGW) modes 
can be produced with similar amplitudes and thus comparable contribution to the CMB anisotropy,

$\left(\frac{\Delta T}{T}\right)_{10^{0}}^{2}=S+T$

The second reason comes from observations. If the scalar perturbation spectrum is 'blue' $\left(n_{S}>1\right)$ then a non-zero $\mathrm{T} / \mathrm{S}>0$ is required to reconcile the $\mathrm{COBE} \Delta T / T$ measurement with the galaxy cluster abundance (Lukash et al., 2000).

The problem of $\mathrm{T} / \mathrm{S}$ is fundamental but can be treated at the moment only theoretically. A serious discussion on the observational detection of $\mathrm{T} / \mathrm{S}$ could be launched after CMB polarization measurements, which would require the instrumental sensitivity $\sim 1 \mu K$ currently marginally reachable.

\section{CONCLUSIONS AND TENDENCIES}

As never before, cosmologists are close to recovering the real model of our Universe and the postrecombination CDP spectrum directly from observations, both $\Delta T / T$ and LSS, and to creating an exciting link to the physics of the very early Universe. We are going to gain the data from the advanced ground and space based CMB explorers as well as large surveys of spatial distribution of galaxies, galaxy clusters and quasars, to delimit the cosmological model with unprecedented precision.

The list of current conclusions may be incomplete:

- dark energy is not curvature $\left(\Omega_{\kappa} \sim 0\right)$;

- quintessence is consistent with $\Lambda$-term;

- defects are not responsible for LSS formation;

- the slope of the fundamental CDP spectrum is consistent with Harrison-Zel'dovich one $\left(n_{S} \simeq 1\right)$;

- distant supernovae Ia indicate the current acceleration of the Universe $\left(\Omega_{m}<0.5\right)$;

- $\Delta T / T$ and LSS data hint on low $\Omega_{m} \lesssim 0.5$;

- baryonic dark matter is indicated by the $\Delta T / T$ acoustic peaks $\left(\Omega_{b} h^{2} \simeq 0.03\right)$;

- adiabatic $\Lambda$ CDM models has survived $\left(\Omega_{m} \sim 0.3, \Omega_{\Lambda} \sim 0.7\right)$;

- the best-fit $\Lambda \mathrm{MDM}$ models predict half-to-half matter/energy domination $\left(\Omega_{m} \sim 0.5, \quad \Omega_{\Lambda} \sim 0.5\right)$ (Novosyadlyj et al., 2000).

In view of current cosmological discussion we can happily state a common observational agreement on four important points:

- $\Omega_{t o t}=1 \pm 0.1$ (we live in a flat Friedmann Universe);

- $n_{S}=1 \pm 0.1$ (the spectrum of density perturbations is scale free);

- $h=0.65 \pm 0.1$ (the Hubble constant is close to $65 \mathrm{~km} \mathrm{~s}^{-1} \mathrm{Mpc}^{-1}$ );

- the observed spectrum of density perturbations is best fitted by $\Lambda \mathrm{CDM}\left(\Omega_{m} \sim 0.3, \Omega_{\Lambda} \sim 0.7\right)$ and $\Lambda \operatorname{MDM}\left(\Omega_{m} \sim 0.5, \Omega_{\Lambda} \sim 0.5\right)$ models;

Nevertheless, the discussion about baryonic and matter abundances in the Universe is up on the agenda. The critical tests in building up the true cosmology are the following:

- direct detection of the neutrino rest masses and other forms of dark matter;

- determination of the locations and amplitudes of the acoustic peaks in $\Delta T / T$;

- detection and investigation of the polarization of $\Delta T / T$; 
- further progress in data analysis of the formation and evolution of large scale structure in the Universe;

- development of the geometrical tests (supernovae Ia, $h$, light elements abundance).

Summarizing, we can state a prominent progress: the detalization and status of some parts of cosmology remind one an enginearing science level. Still the fundamental cosmological issues waiting their solutions remain the following:

- the problem of dark matter (multicomponent, baryonic, ...);

- the problem of $\Lambda$-term (value, the period of domination, ...);

- the break in the CDP spectrum slope at scale $\sim 100 h^{-1} \mathrm{Mpc}$ which requires new physical explanation (scale, physics of dark matter, why LSS and CMB scales are close to each other $\left(\sim 100 h^{-1} \mathrm{Mpc}\right)$ );

- the intensity of cosmic gravity waves ( $\mathrm{T} / \mathrm{S}$, energy scale of cosmic inflation).

\section{ACKNOWLEDGEMENTS}

The work was supported in part by INTAS (97-1192). The author is grateful to the Organizing Committee for the hospitality.

\section{REFERENCES}

Bennett, C.L., Banday, A.J., Gorski, K.M., Hinshaw, G., Jackson, P., et al., ApJ, 464, L1, 1996.

Boyle, B.J., Terlevich, R.J., MNRAS, 293, L49, 1998.

Einasto, J., Einasto, M., Gottlöber, S., Müller, V., Saar, V., et al., Nature, 385, 139, 1997.

Guzzo, L., ApJ, 382, L5, 1991.

Jaffe, A.H., Ade, P.A.R., Balbi, A., Bock, J.J., Bond, J.R., et al., astro-ph/0007333.

Juszkiewicz, R., Bouchet, F.R., astro-ph/9602134.

Komberg, B.V., Kravtsov, A.V., Lukash, V.N., MNRAS, 282, 713, 1996.

Komberg, B.V., Lukash, V.N., MNRAS, 269, 277, 1994.

Lukash, V.N., Mikheeva, E.V., Gravitation \&6 Cosmology, 2, 247, 1996.

Lukash, V.N., Mikheeva, E.V., Int.J.Mod.Phys.A, 15, 3783, 2000.

Lukash, V.N., Mikheeva, E.V., Muller, V., Malinovsky, A.M., MNRAS, 317, 795, 2000.

Mazure, A., Katgert, P., den Hartog, R., Biviano, A., Dubath P., et al., A\&AA, 310, 31, 1996.

Novosyadlyj, B., Durrer, R., Gottloeber, S., Lukash, V.N., Apunevych, S., A $\dot{\xi}$ A, 356, 418, 2000.

Peacock, J., astro-ph/9608151.

Perlmutter, S., Aldering, G., Della Valle, M., Deustua, S., Ellis, R.S., et al., Nature, 391, 51, 1998.

Watson, A., Science, 278, 574, 1997. 